Mots. Les langages du politique

Chiffres et nombres dans l'argumentation politique

\title{
Le chiffre au service du droit ou le droit au service du chiffre?
}

Rachel Vanneuville

\section{OpenEdition}

1 Journals

Édition électronique

URL : https://journals.openedition.org/mots/20985

DOI : $10.4000 /$ mots. 20985

ISSN : 1960-6001

Éditeur

ENS Éditions

Édition imprimée

Date de publication : 15 décembre 2012

Pagination : 123-136

ISBN : 978-2-84788-387-9

ISSN : 0243-6450

\section{Référence électronique}

Rachel Vanneuville, "Le chiffre au service du droit ou le droit au service du chiffre ? », Mots. Les

langages du politique [En ligne], 100 | 2012, mis en ligne le 15 décembre 2014, consulté le 22 avril 2022. URL : http://journals.openedition.org/mots/20985; DOI : https://doi.org/10.4000/mots.20985 


\section{Le chiffre au service du droit ou le droit au service du chiffre?}

8100 textes législatifs, 360000 règlements, 2223 pages au Code du travail, plus $50 \%$ de décrets en 10 ans... Extraits d'articles de quotidiens nationaux ${ }^{1}$, ces exemples de chiffres relatifs à la production des textes de droit pourraient être multipliés, tant il est devenu fréquent, depuis une vingtaine d'années, d'évoquer le droit sous cette forme. Si nous ne pouvons dater précisément, dans l'état actuel de nos recherches, la naissance de cette «mise en chiffres» du droit, il est toutefois possible de repérer un moment important de sa mise en circulation dans les discours publics - médiatiques et politico-administratifs : la parution en 1992 du Rapport annuel 1991 du Conseil d’État consacré à la sécurité juridique (Conseil d’État, 1992). Il propose des mesures chiffrées de la "dégradation de la norme » (ibid., p.7) et va devenir une référence sur la question (Vanneuville, 2010). S'intéressant à l'opération de chiffrage de la justice, Antoine Vauchez (2008) note qu'elle a engendré des luttes autour de l'interprétation des chiffres, renvoyant à des enjeux de pouvoir relatifs au gouvernement de l'institution judiciaire. En ce qui concerne le chiffrage du droit sous sa forme textuelle, point de luttes de ce type. Plus que cela : alors que le Conseil d'État reconnaît l'imprécision de ses mesures, elles sont néanmoins reprises comme si leur fiabilité importait peu. Ce n'est qu'en 2003 que paraît une étude exclusivement consacrée à ce chiffrage, qui contribue à mettre en lumière son caractère discutable (Grass, 2003). Commentant cet article, le président de la Commission des lois de l'Assemblée nationale reconnaissait que le nombre et le volume des lois en vigueur étaient difficilement appréciables et que les résultats du comptage étaient en effet «aléatoires». Il concluait néanmoins : «Ce que l'on peut affirmer avec certitude, c'est que notre pays connaît sur ce plan une inflation en nombre comme en volume » (Blanc, 2003, p. 2977-2978). Ainsi, les mesures produites ne sont pas fiables mais elles autorisent néanmoins à affirmer «avec certitude » qu'il y a eu dégradation. On pourrait multiplier les citations d'auteurs qui adoptent cette argumentation.

1. Thierry Desjardins, "L'insupportable dictature des lois», Le Figaro, 10 janvier 2000 ; Éric Rohde, «"Trop de loi tue la loi”... La jungle législative», Le Monde, 24 janvier 2007.

CNRS, Triangle,Lyon 2

rachel.vanneuville@ens-lyon.fr

Mots. Les langages du politique $\mathrm{n}^{\circ} 100$ novembre $2012 \bullet 123$ 
Comment comprendre ce qui fonde la «valeur sociale »² de cette opération de chiffrage du droit, et le consensus qui s'exprime à son sujet? C'est à cette question que l'article propose de répondre, en analysant les discours qui décrivent depuis vingt ans l'inflation normative française3. Il s'agira de s'interroger, d'une part sur la construction sociale de l'autorité de ces chiffres, en montrant notamment le rôle de leur principal producteur : le Conseil d'État; d'autre part sur la manière dont l'usage de ces chiffres a permis de mobiliser un ensemble d'acteurs autour de la réforme du système d'élaboration des normes; enfin sur la vision du droit qui est promue à travers le recours aux chiffres. Alors que l'objectif des discours sur l'inflation normative est de revaloriser le rôle social du droit, l'opération de chiffrage semble avoir contribué à la banalisation du droit et favorisé sa soumission à des impératifs managériaux.

\section{La mise en chiffres du droit}

La critique de la prolifération des textes est ancienne : nombre d'auteurs contemporains le soulignent, citant entre autres Montaigne, Montesquieu et, pour la période plus récente, les études, érigées en référence, de René Savatier ("L'inflation législative et l'indigestion du corps social», 1977) et de Jean Carbonnier ("L'inflation des lois», 1979). Le propos n'est pas ici d'effectuer une socio-histoire de cette thématique - qui reste à faire - mais de constater qu'elle resurgit avec force dans les années quatre-vingt, qu'elle va mobiliser un ensemble d'acteurs et générer des réformes du travail administratif et parlementaire. Autant d'éléments qui invitent à s'interroger sur les conditions de possibilité de ce «succès », à comprendre, en paraphrasant Juliette Rennes, comment le chiffrage a pu permettre aux acteurs de réinvestir «cette longue durée des discours et des arguments » et leur permettre de déplacer ce qui est «pensable, dicible et réalisable» (Rennes, 2011, p.152).

\section{Réforme de l'État et positionnement du Conseil d'État}

Le contexte dans lequel s'inscrit le Rapport 1991 du Conseil d'État est celui d'une montée en puissance des discours sur la réforme de l'État, le tournant des années quatre-vingt-dix se caractérisant par l'institutionnalisation de pro-

2. Selon l'expression d'Albert Ogien (2010, p. 21, p. 20) pour lequel «la valeur sociale du chiffre ne tient pas à ce qu'il est une représentation certifiée et concise d'une réalité objective», mais à l'importance dont il est doté par les participants à une forme d'action politique.

3. Les discours désignent ici les rapports et allocutions publics (rapports du Conseil d’État, rapports parlementaires, allocutions publiques de responsables politiques et de professionnels du droit), les articles de revues juridiques. Les quotidiens ont été également sondés: Le Monde depuis 1987, et par l'intermédiaire de la base de données Factiva, quelques autres publications (Le Figaro, Libération, Les Échos...). 
grammes de réforme administrative (Bezes, 2009). Comme le met en lumière un ensemble d'articles publiés par Jacques Caillosse à cette époque, c'est à « une mise en doute du droit administratif que travaille aussi la réforme administrative » (Caillosse, 1989, p. 3), droit accusé d'être un frein à la modernisation de l'État. En particulier, la question de la prolifération des normes et de leur caractère obscur est l'un des principaux arguments dans la critique de la lenteur et de l'opacité de l'administration. Considéré lui-même comme un «symbole des privilèges d'une administration [jugée] trop puissante » (Lochak, 1993, p. 142), le Conseil d'État fait l'objet de fortes critiques et entreprend une «stratégie de relégitimation» (ibid., p.146).

C'est dans ce cadre qu'il faut comprendre la publication du rapport sur l'insécurité juridique : il manifeste le souci de la Haute assemblée de s'approprier le discours sur le droit afin de ne pas en laisser le monopole à ses détracteurs publics et de revendiquer dans le même temps sa capacité à participer aux politiques de réforme administrative4. La mise en chiffres du "dérèglement» normatif à laquelle le rapport procède accrédite ainsi la véracité des discours dénonçant l'hyper-réglementation tout en attestant de la légitimité du Conseil à être un lieu d'expertise légitime sur la question. D’abord parce qu'il serait un lieu privilégié de production et de recueil de l'information relative au droit : il est expliqué dans le rapport que les données proviennent de l'activité des sections administratives 5 mais également de diverses études que le Conseil a menées grâce à ses relations privilégiées avec les ministères et avec le Secrétariat général du gouvernement. Ensuite parce que sa mission de « gardien de la cohérence du droit » (Conseil d’État, 1992, p. 7) le rendrait particulièrement habilité à agir sur ce dérèglement. C'est ici plus spécifiquement la fonction de bureau d'étude du Conseil qui est mise en avant, la Constitution conférant à l'institution la mission d' " attirer l'attention des pouvoirs publics sur les réformes d'ordre législatif, réglementaire ou administratif qui lui paraissent conformes à l'intérêt général ». Le Rapport annuel est l'un des supports de ces propositions de réforme, et va devenir dans les années quatre-vingt l'un des moyens de faire connaître, et reconnaître, cette fonction de l'institution. Le Rapport 1991 s'inscrit dans cette démarche communicationnelle: «Si nous voulons être lus d'un public plus large que notre public actuel nous devons rester très concrets et, surtout, être lisibles », préconise Françoise Jurgensen, responsable de son écriture6 ${ }^{6}$. Le choix du contentieux fiscal comme illustration des changements incessants de

4. Analysant les rapports annuels du Conseil d’État depuis 1991, J. Caillosse note ainsi : le Conseil "souligne défaillances, ambiguïtés et autres incohérences du droit partout où elles existent, pour mieux se donner les moyens d'une remise en ordre dans tel ou tel champ de l'action publique» (Caillosse, 2010, p. 131).

5. La Constitution de 1958 attribue compétence au Conseil d’État, via ses sections administratives, pour donner des avis sur certains projets de décrets, sur les projets d'ordonnance et les projets de loi.

6. F. Jurgensen, « Note pour le Vice-Président », 12 juin 1991, Archives du Conseil d'État, Centre d'archives contemporaines, cote $20080196 / 43$. Souligné dans le texte. 
la règle de droit est ainsi en partie effectué parce qu'il s'agit « d'un thème très "porteur" sur le plan médiatique et qui intéresse [...] un grand nombre de Français »7. La mise en chiffres de l'insécurité juridique participe de cette stratégie : elle simplifie le message - l'insécurité juridique va notamment prendre la forme du «trop de... » - et facilite sa circulation : les évaluations effectuées par le Conseil seront incessamment reprises - dans les médias, la littérature juridique, les discours politiques. Le chiffrage du droit auquel procède le Conseil d’État en 1991 est donc fortement lié à des enjeux de positionnement de la Haute assemblée dans le paysage institutionnel et dans l'espace public de l'époque. Une partie de la valeur du chiffre réside dans sa production même, qui offre au Conseil un moyen supplémentaire d'afficher sa qualité d'expert en matière de contrôle du droit : la proposition faite, en conclusion du rapport, que soit attribuée au Conseil ${ }^{8}$ la fonction d'observer les flux et les stocks normatifs n'est qu'une manière d'expliciter ce qui est déjà à l'œuvre. Une autre partie de son importance tient dans le surcroît argumentaire qu'offre le chiffre pour décrire la dégradation normative.

\section{Donner du poids aux mots}

Le chiffrage peut en effet être ici analysé comme un moyen «d'exprimer et de faire exister sous une forme numérique ce qui, auparavant, était exprimé par des mots » (Desrosières, 2008, p. 10), autrement dit un moyen de donner une dimension supplémentaire aux discours préexistants en les renforçant par des données chiffrées. Dans le Rapport, celles-ci visent à embrasser l'ensemble de la production normative. Sont ainsi quantifiés le nombre de lois votées depuis les débuts de la Cinquième République, leur accroissement annuel moyen ainsi que celui des décrets; la hausse du volume des textes, exprimé en augmentation du nombre moyen de lignes que comportent les lois, du nombre de pages du Journal officiel de la République et du Journal officiel des communautés; le volume des formulaires auxquels sont soumis les usagers, matérialisé à la fois par l'évaluation de la longueur qu'ils représentent en étant mis bout à bout - une entreprise moyenne ordinaire doit ainsi remplir 3,6 mètres de formulaires (Conseil d'État, 1992, p.19) - et par l'augmentation du nombre de pages de la notice explicative accompagnant le formulaire de déclaration de revenus, exprimée en pourcentage ( $+600 \%$ entre 1980 et 1990$)$.

De nombreux autres chiffres sont encore mentionnés dans le rapport, ayant trait à des lois et des domaines législatifs particuliers. Leur accumulation, asso-

7. F. Jurgensen, «Préparation du rapport annuel 1991. Compte-rendu de la réunion du 17 avril $1991 »$, Archives du Conseil d'État, Centre d'archives contemporaines, cote 20050574/10.

8. Ou aux services du Premier ministre, ajoute le rapport, désignant par là le Secrétariat général du gouvernement, dont la direction est traditionnellement assurée par un membre du Conseil d’État (Conseil d’État, 1992, p.47). 
ciée à la diversité des formes de quantification - nombre de lignes, de mètres, pourcentages, moyennes... - a pour effet de donner à voir le caractère multiforme et étendu du phénomène de dégradation normative, voire son caractère... non mesurable. Ceci peut sembler paradoxal dans le cadre d'une entreprise destinée à objectiver la quantité de textes normatifs et son évolution. Cela l'est moins si l'on considère que la valeur du chiffrage réside moins dans la précision apportée à la connaissance du phénomène que dans son caractère symbolique : mettre les mots décrivant l'inflation normative en nombres permet de donner une épaisseur supplémentaire aux premiers, et réciproquement. En l'occurrence, la mise en chiffres proposée permet de justifier l'usage des expressions : «marée normative», «prolifération des textes», « effervescence normative », «droit bavard », «logorrhée législative et réglementaire», autant d'expressions qui vont faire florès. De la même manière, c'est parce que les chiffres livrés dans le rapport sont associés à ces expressions qu'ils prennent sens : dire que le nombre de lois a augmenté ou que le nombre de leurs lignes s'est accru ne devient signe de dégradation que parce que ces nombres sont reliés à des mots stigmatisants. Autrement dit, chiffres et mots se renforcent mutuellement, et sont destinés à faire passer le cœur du message du Rapport 1991 : c'est l'État de droit, donc la démocratie, qui sont menacés par cette profusion textuelle.

Bien qu'aléatoire, le chiffrage peut ainsi générer la certitude du diagnostic de la dégradation de la norme ou, plus exactement, servir à conforter une certitude qui préexistait à son usage. Si le Rapport devient une référence sur ce sujet, c'est en grande partie parce qu'il est une chambre d'enregistrement des critiques existantes envers le droit français tout en leur permettant de prendre une ampleur supplémentaire. Dans ce cadre, la véracité du chiffre importe peu. Sa valeur sociale réside dans le fait que l'on peut dorénavant ajouter des chiffres aux mots et, surtout, faire prendre du poids à ces derniers. Le recours aux chiffres va ainsi progressivement devenir l'une des formes privilégiées pour dénoncer le «désordre normatif» (Revue du droit public, 2006).

\section{L'expansion des chiffres du droit}

Les chiffres du Rapport 1991 vont en effet circuler dans les espaces médiatique, politique et administratif. Cette circulation peut certainement être rapportée à un phénomène plus général de «croyance» dans la vertu des chiffres comme mode d'objectivation d'une réalité sociale. Elle s'ancre plus spécifiquement dans l'intérêt qu'ont des acteurs de ces espaces à se les approprier pour faire valoir leurs propres objectifs. Ce faisant, ils vont également contribuer à nourrir l'opération de chiffrage. 


\section{Circulation des chiffres et accroissement des instruments de mesure}

La circulation des chiffres du Rapport 1991 s'opère par plusieurs canaux. La production de rapports relatifs au travail parlementaire et gouvernemental d'une part, et au travail administratif d'autre part - soit au sujet des deux lieux désignés comme principaux responsables de la surproduction normative - en est un. Parlementaires comme hauts fonctionnaires font fructifier le discours sur l'inflation des normes pour proposer des réformes de l'activité d'élaboration des textes, qu'ils soient législatifs ou réglementaires. Pour les parlementaires, l'enjeu est essentiellement de promouvoir la revalorisation de la loi dont ils sont les représentants (par ex. : Bignon, Sauvadet, 1995; Quilès, 2006)9; pour les hauts fonctionnaires, c'est avant tout la réforme de l'État qui est en jeu, l'amélioration de la qualité réglementaire étant décrite comme participant à l'avènement d'un État plus «responsable», plus «accessible» pour les citoyens (par ex. : Picq, 1995; Mandelkern, 2002). Nombre de ces rapports répercutent également les doléances des acteurs économiques à l'encontre de la complexification des textes, qui nuirait à la compétitivité des entreprises (par ex. : Bignon et Sauvadet, 1995; Mandelkern, 2002). Quant aux juristes, universitaires et praticiens, ils s'approprient d'autant plus facilement la question qu'elle touche au cœur de leur activité quotidienne : commenter et mettre en cohérence le corpus normatif, le contrôler et le mettre en action ${ }^{10}$. La fortune de la thématique de l'inflation normative se manifeste, et s'amplifie, à travers les prises de parole publiques d'hommes politiques ou de hauts fonctionnaires, qui sont largement relayées par la presse (par ex. : «La prolifération des lois», Le Figaro, 20 septembre 2000; «ll faut concentrer la loi sur l'essentiel», Libération, 18 janvier 2005) ${ }^{11}$. Sans pouvoir détailler ici l'ensemble de ces discours, toujours est-il qu'ils indiquent que chiffres et mots sur le droit s'apparentent à un « dispositif d'intéressement» (Callon, 1986) qui favorise une alliance entre divers acteurs - politiques, administratifs, économiques, juridiques, médiatiques - aux intérêts hétérogènes. Surtout, cette circulation des chiffres va leur permettre de proliférer.

Si de nombreux rapports des années quatre-vingt-dix se contentent de reprendre les données du Conseil d’État (par ex. : Picq, 1995; Rufin, 1996), d'autres les étoffent et sophistiquent leur présentation, tel le rapport des parlementaires Jérôme Bignon et François Sauvadet (Bignon et Sauvadet, 1995) qui

9. Sur les usages politiques, par les parlementaires, du thème de l'inflation normative, voir Thomas, 2008.

10. Pour une bibliographie de leurs écrits, voir par exemple Drago, 2005.

11. Pour un aperçu des interventions médiatiques d'hommes politiques dans les années quatrevingt-dix, voir Grass, 2003. Sur l'appétence des médias à se saisir des chiffres, voir Vauchez, 2008 , p. 117. 
propose des tableaux, graphiques et camemberts et qui ajoute des données relatives aux délais d'élaboration des lois, à la publication des décrets d'application, etc. Il n'est pas le seul (par ex. : Mercier, 2000; Mandelkern, 2002). Autrement dit, aux chiffres donnés par le Conseil d'État en 1991 vont s'en ajouter d'autres - le poids du Journal officiel est mentionné dans un entretien que donne au Figaro le président de l'Assemblée nationale ("Jean-Louis Debré : “La loi doit se limiter à édicter des normes” ", Le Figaro, 16 janvier 2006). En 2006, le Conseil d'État consacre de nouveau son rapport public à l'insécurité juridique (Conseil d'État, 2006), ce qui va contribuer à accélérer la mise en chiffres. Justifiant la production de ce rapport par «l'aggravation du problème», le Conseil d'État lance à cette occasion un nouveau mode de communication en proposant sur son site internet un dossier synthétique destiné à la presse, dossier dont les chiffres seront repris dans de nombreux journaux, avec une tonalité dramatique (voir encadré).

\section{La reprise des chiffres du rapport 2006 dans la presse : quelques exemples}

" “Aux 9000 lois et 120000 décrets recensés en 2000 sont venus s’ajouter, en moyenne, 70 lois, 50 ordonnances et 1500 décrets par an”, relève le Conseil d'État. De plus, environ $10 \%$ des articles des 59 codes changent chaque année. Un constat "ubuesque" dans une République où nul n'est censé ignorer la loi.» ("Conseil d’État : il y a trop de lois en France », Le Parisien, 15 mars 2006)

«Le rapport de Josseline de Caussade [rédactrice du Rapport 2006], s’il est édifiant et montre à quel point notre pays possède tous les "atouts" pour décrocher la médaille d'or de l'inflation législative, ne va pas attirer à son auteur que des sympathies. En effet, la rapporteuse [...] ne s'arrête pas à la description de cette avalanche qui ensevelit juges, citoyens, entreprises et collectivités locales un peu plus tous les ans sous des tonnes de textes. Elle ne s'arrête pas à la mesure que prennent des projets de lois de plus en plus complexes et de plus en plus longs : 218 pages pour la loi sur la politique de santé de $2004 ; 231$ pages pour celle sur les libertés et responsabilités locales. Sans compter que cette furia légiférante, cette hystérie décrétante, cette obésité ordonnante contribue de surcroît à la déforestation : des années 1980 à aujourd'hui, le Journal Officiel est passé de 15000 à 23000 pages! On comprend que le rapport du Conseil d’État en tire la conclusion que ce système est source d'“insécurité juridique", mais qu'il représente également une "menace de l’État de droit” » ("L'inflation législative, un mal français... », La Charente libre, 16 mars 2006) «Des chiffres alarmants :

- 9000 lois, 120000 décrets recensés en 2000. Depuis, sont venus s'y ajouter, en moyenne, chaque année, 70 lois, 50 ordonnances, 1500 décrets [...].» ("Le Conseil d'État appelle les politiques à stopper l'inflation législative», Les Échos, 15 mars 2006)

La production de données sur les textes est facilitée par les réformes liées à la thématique de l'inflation normative : en 1996 est créé l'office parlementaire d'évaluation de la législation, «chargé de rassembler des informations et de procéder à des études pour évaluer l'adéquation de la législation aux situations 
qu'elle régit » (loi du 14 juin 1996) ; un ensemble de circulaires relatives à l'amélioration de la qualité de la réglementation insistent, depuis 1995, sur la mise en place d'indicateurs et d'instruments de connaissance des textes existants. Suite à la publication du rapport 2006 du Conseil d’État, le site Légifrance crée au printemps 2006 une rubrique «Évolution du volume des textes», destinée à compléter «l'information de ses utilisateurs par la diffusion de statistiques sur l'évolution quantitative du droit français » et permet notamment «d'accéder à une représentation graphique de l'évolution [des] codes ${ }^{12}$. À partir de 2007 , le Secrétariat général du gouvernement développe « un système d’information permettant d'évaluer de façon continue les caractéristiques de la production normative ॥ $^{13}$. Il en résulte la publication, en janvier 2011 , d'une brochure qui met en chiffres, tableaux, graphiques ou camemberts le stock de textes en vigueur, l'évolution du nombre de lois et de leurvolume - en nombre d'articles et de mots -, le nombre annuel de modifications apportées aux codes... (Secrétariat général du gouvernement, 2011). Bref, de nombreux instruments sont mis en place, qui vont permettre aux chiffres d'être actualisés, étoffés et diffusés, nourrissant la dramatisation des discours qui les mobilisent.

Évoquant «l'inflation des chiffres mesurant l'inflation des lois», le juriste Hervé Moysan (2007) résume bien l'accroissement des mesures qui s'est produit depuis le Rapport 1991. Il remarque également qu'elles ont été acceptées sans discussion tant par la doctrine que par les hommes politiques ${ }^{14}$, citant pour seule étude critique approfondie celle d’Étienne Grass (2003). Un bref arrêt sur cette étude permet de prendre la mesure du caractère d'évidence qu'ont pris les discours sur l'inflation normative.

\section{La dégradation des normes comme discours d'évidence}

L'intérêt de l'article d'E. Grass tient dans ce qu'il ouvre la «boîte noire » du chiffrage de l'inflation normative, en discutant les indicateurs choisis. Ainsi du nombre de pages du Journal officiel qui ne tient pas compte des diverses rubriques dont s'est étoffé le périodique (décisions du juge constitutionnel, traités annexés aux lois d'autorisation...) : un indicateur qu'il décrit comme non pertinent pour conclure à une inflation normative. De la même manière, la loi serait « une mauvaise unité de compte pour l'établissement d'une série statistique» (ibid., p.144) car, entre autres, «son périmètre n'est pas homogène» (ibid., p.146). Comptant néanmoins le nombre de lois promulguées

12. Suivant la présentation de cette rubrique sur le site Légifrance : http://www. legifrance. gouv. $\mathrm{fr} / \mathrm{html} /$ statistiques_normatives/liste_stats. htm (consulté le 11 juin 2007).

13. "Avis de vacance d'un emploi de directeur de projet»: http://admi.net/jo/20070301/ PRMX0710091V.html (consulté le 16 mars 2007).

14. Il existe des opinions critiques quant à cette opération de chiffrage, mais elles sont peu nombreuses et, surtout, n'ont guère d'écho. Voir Moysan, 2002, 2007, 2010 ; Mbongo, 2005; Rolin, 2006. 
depuis 1968, il montre que leur nombre moyen annuel a diminué de $20 \%$, alors que le Conseil d'État évoquait un accroissement annuel de $35 \%$ : ce sont ici les dates de références choisies pour le dénombrement qui sont discutées. Quant à l'allongement des lois par la multiplication de leur nombre d'articles, E. Grass le relativise en montrant qu'il ne faut pas prendre globalement le chiffre des articles mais les rapporter à chaque loi : ainsi, en 2000, $62 \%$ des lois comportent moins de 10 articles et seules $5 \%$ (soit 3 lois) ont plus de 100 articles. Sans entrer plus avant dans les détails de la démonstration, elle indique que la mise en chiffres est sujette à controverses. Or non seulement elle ne l'est pas, mais les mêmes indicateurs continuent à être utilisés et sophistiqués - comptage du nombre de mots des lois ou des occurrences de tel ou tel terme par exemple-, comme si discuter de l'opération de chiffrage revenait à mettre en doute le «constat» qu'elle charrie : le système normatif continue de se dégrader. Ne pas discuter des chiffres permet ainsi de fermer les discours.

En donnant à voir l'hétérogénéité des éléments mesurés, l'article d'E. Grass présente un autre intérêt : il invite à poser la question du sens du comptage.

\section{Le droit saisi par le chiffre}

Entrant dans la matière juridique et la manière dont elle est quantifiée, E. Grass donne implicitement à voir ce qu'Alain Desrosières nomme les «conventions» qui sont au fondement de toute activité de quantification : pour pouvoir mesurer, il faut d'abord s'accorder sur ce que l'on mesure (Desrosières, 2008, p.10-11). Il nous semble que c'est une conception particulière du droit qui est révélée par les mesures dont il est l'objet, un droit qui doit être épuré pour conserver son autorité sociale. Si l'opération de quantification est au service de cet objectif, elle semble pourtant favoriser la banalisation de l'outil juridique.

\section{Du droit «pur»...}

En additionnant nombre de lois, d'articles ou de mots... l'opération de chiffrage fait comme si une loi valait une autre loi, un article un autre article, etc. Autrement dit, tout texte de droit - et ce qui le compose - importe et doit être pris au sérieux. Mais il a d'autant plus de valeur qu'il est rare et sobre : pourquoi mettre ensemble, car c'est ainsi qu'ils sont le plus souvent présentés, les chiffres concernant les articles, mots, lois, variations... si ce n'est pour faire émerger le mot qui peut résumer cet agrégat : «trop »? L’accord qui paraît lier les protagonistes du chiffrage du droit, et qui expliquerait que les mesures ne soient pas questionnées, semble résider dans une vision commune de la «bonne » règle de droit : concise et impérative, conforme à une conception formelle du droit. Selon cette dernière, la règle est le produit de la volonté d'un 
législateur rationnel, volonté exprimée clairement, dans des conditions et dans des formes juridiquement irréprochables, une règle dont la qualité assure sa capacité à organiser le monde social (Corten, 2002, p.199-200), et dont la loi est, en France, l'incarnation historique (Chevallier, 1990). Cette vision se manifeste dans la référence omniprésente aux «préceptes» de Portalis, l'un des pères du Code civil de 1804 : «De bonnes lois civiles sont [...] la source des mœurs»; «la loi permet, ordonne ou interdit »; "l'office de la loi est de fixer, par de grandes vues, les maximes générales du droit [...] et non de descendre dans le détail des questions qui peuvent naître sur chaque matière ». Même si les discours font état de la complexification du monde, de la croissance des droits infranational, européen et international qui sont autant de facteurs d'expansion et de complexité des textes, une vision nostalgique du droit semble leur fond commun ${ }^{15}$. L'opération même de quantification des textes matérialise ainsi un accord sur ce que doit être le droit, tout en indiquant les moyens d'en restaurer la «pureté» : ramener à la «raison juridique » le législateur, une raison porteuse de généralité et de neutralité qui s'opposerait au travail politique, pragmatique, sectoriel et contingent, désigné comme responsable du foisonnement et du désordre textuels (Commaill, Lascoumes, 1995) ${ }^{16}$. Pour autant, l'opération de comptage est fondamentalement ambiguë : si elle sert à dénoncer la démesure des producteurs de textes pour les ramener à la sobriété, elle fait aussi entrer les chiffres dans la matière juridique et, avec eux, une logique gestionnaire (Ogien, 1995).

\section{... au droit instrumental}

La mise en chiffres du droit est essentiellement pensée en termes économiques. S’il existe plusieurs registres de métaphores pour parler de la dégradation des normes - ceux de la catastrophe naturelle (marée, avalanche ) ou de la pathologie médicale (obésité, boulimie ) -, c’est le registre économique qui prédomine depuis les années quatre-vingt-dix, avec la description du phénomène en termes d'inflation. Comme l'écrivait Jean Carbonnier en 1979 (p. 272) : " une inflation, très sèchement, ça doit pouvoir se chiffrer », indiquant bien la relation au chiffre induite par l'usage de cette métaphore. Celle-ci conduit à parler du droit comme d'un «produit» dont la «surproduction» conduit à la «dévaluation » de sa valeur (Conseil d’État, 1992, p.16, p. 20); à évoquer les «stocks et flux» des lois qu'il faut gérer (ibid., p. 19), bref, à penser l'élaboration du droit sous la forme d'un circuit de production dont il s'agit de contrôler la qualité et de rentabiliser le fonctionnement. Parmi les chiffres qui servent

15. Certains auteurs le remarquent, par exemple : Deumier, $2005 a$.

16. Est par exemple critiquée la soumission des responsables politiques à un impératif communicationnel qui les conduirait à multiplier les lois circonstancielles (Conseil d’État, 2006, p. 254 et suiv.). 
à quantifier le droit se glissent aussi ceux qui évaluent son coût. Le Rapport 1991 mentionnait déjà le «coût financier » du temps passé par les entreprises à remplir les formulaires administratifs ainsi que le «coût de gestion » que ces derniers représentent pour l'administration chargée de les dépouiller (ibid., p. 19). C'est cependant dans les discours plus récents que le chiffrage du coût est plus systématiquement associé aux normes (Lasserre, 2004; Delaunay et al., 2010, p. 670), et que sont mises en avant les notions de «prospective» et de «pilotage » de l'activité normative (Gilberg, 2007, p. 291 et suiv.). Ces préoccupations d'efficience économique et sociale doivent être replacées dans un contexte plus large qui contribue à transformer le sens du chiffrage.

La saisie du droit par le chiffre est en effet à l'œuvre tant au niveau européen qu'au niveau international dès les années quatre-vingt-dix. "La recherche d'une meilleure compétitivité des systèmes productifs a conduit [l'OCDE comme la Commission européenne] à s'interroger - dans le but de les maîtriser - sur les coûts et les charges liés à "l'environnement réglementaire" » (Mandelkern, 2002, p. 6). C'est dans les années deux mille que ces préoccupations extra-nationales commencent à circuler dans les discours français, mais l'impact de l'approche économique du droit qui les sous-tend prend véritablement de l'ampleur après la parution, en 2004, du Rapport Doing Business de la Banque mondiale. Celui-ci met en cause le manque d'attractivité économique du droit français et génère des réactions assez vives en France. Il conduit notamment à la mise sur pied, sur l'impulsion d'un membre du Conseil d'État, d'un groupe de recherche sur l'attractivité économique du droit, qui va s'attacher à discuter les indicateurs et la méthodologie utilisée par la Banque mondiale (Du Marais, 2006a). Pour autant, le fond du rapport n'est pas mis en cause : évaluer les performances du droit français et se soucier de ses effets économiques est l'un des objectifs à poursuivre (Du Marais, 2006b). Ces préoccupations semblent d'autant mieux intégrées qu'elles s'inscrivent dans le processus de réforme managériale de l'État qui s'amplifie à partir des années deux mille (Bezes, 2009, p. 421 et suiv.) ${ }^{17}$. La Loi organique relative aux lois de finances de 2001 est ainsi présentée comme constituant une «forte incitation » à développer les calculs des coûts de la réglementation, et à envisager les règles en termes de performance (Lasserre, 2004, p. 17-18). De la même manière que le développement d'une approche économique du droit a conduit à penser la justice comme "une unité économique "comme les autres" (Vauchez, 2008, p. 117), elle conduit à faire du droit un instrument de l'action publique «comme les autres». Dans ce contexte général, la mise en chiffres du droit engagée depuis les années quatre-vingt-dix, et la rationalisation des outils destinés à l'évaluer (indicateurs mesurant les taux d'application des lois, le coût de production des normes, tableaux de suivi de la production normative...),

17. Sur la pénétration d’une rationalité managériale dans le droit : Chevallier, 1993. 
ont facilité l'emprise des impératifs de performance sur le droit. Si les chiffres servent toujours à dénoncer «l'intempérance » normative, ils sont aujourd'hui des outils d'orientation et de contrôle de l'activité normative.

"Aléatoire », «imprécise » et reconnue comme telle, la mise en chiffres du droit des années quatre-vingt-dix tire sa valeur sociale du crédit supplémentaire qu'elle confère aux discours promouvant la remise en ordre du système normatif. Le quasi-consensus dont elle fait l'objet permet à cette opération de quantification de s'étoffer, contribuant, pour reprendre l'analyse d'A. Desrosières (2008, p.12), à reconfigurer et transformer l'appréhension du droit. Mobilisés pour donner à voir la dégradation formelle du droit et désigner l' «épuration» de ce dernier comme moyen de lui rendre "son honneur perdu» (Deumier, 2005b), les chiffres ont pénétré la matière juridique de toutes parts. Évaluant le nombre de ses mots, son volume, ses rythmes de changement, ses délais de production..., ils ont en quelque sorte contribué à soumettre son «honneur » à des opérations comptables. Le processus continu de chiffrage du droit depuis vingt ans a ainsi facilité sa saisie par une logique gestionnaire, les chiffres servant aujourd'hui à « revaloriser» le droit en permettant l'évaluation de sa production et de ses effets sociaux et économiques ${ }^{18}$.

\section{Références}

BezÈs Philippe, 2009, Réinventer l'État. Les réformes de l'administration française (1962-2008), Paris, PUF.

Bignon Jérôme, SAUVADET François, 1995, L'insoutenable application de la loi, Rapport d'information n²172, Assemblée nationale.

BLANC Étienne, 2003, Assemblée nationale. Débats parlementaires, 3 éance du mardi 8 avril 2003, Journal officiel du 9 avril, p. 2977-2978.

CAIllosse Jacques, 1989, "La réforme administrative et la question du droit », AJDA, 20 janvier, p. 3-8.

- 2010, "Le discours de la réforme administrative. De quelle réforme administrative le rapport annuel du Conseil d’État dessine-t-il les contours?», P. Mbongo, O. Renaudie éd., Le rapport public annuel du Conseil d'État. Entre science du droit et discours institutionnel, Paris, Cujas, p. 125-144.

Callon Michel, 1986, "Éléments pour une sociologie de la traduction. La domestication des coquilles Saint-Jacques et des marins-pêcheurs dans la baie de SaintBrieuc », L'Année sociologique, n³ 36, p.169-208.

CARBONNIER Jean, 1979, Essais sur les lois, Évreux, Répertoire du notariat defrénois.

CheVAllier Jacques, 1990, "La dimension symbolique du principe de légalité », Revue du droit public, nº6, p. 1651-1677.

18. Je remercie les évaluateurs de la revue ainsi que Nicolas Bué pour leurs commentaires de la première version de ce texte. 
- 1993, "La juridicisation des principes managériaux», Politiques et management public, vol. XI, $\mathrm{n}^{\circ} 4$, p. 111-134.

COLLECTIF, 2006, Le désordre normatif, dossier dans Revue du droit public, $\mathrm{n}^{\circ} 1$.

Commaille Jacques, LAscoumes Pierre, avec la collaboration de Fournier Catherine [1995], La production gouvernementale du droit, s.l.n.d.

CONSEIL D'ÉTAT, 1992, Rapport public 1991, Paris, La Documentation française.

- 2006, Rapport public 2006, Paris, La Documentation française.

CORTEN Olivier, 2002, «La persistance de l'argument légaliste. Éléments pour une typologie contemporaine des registres de légitimité dans une société libérale », Droit et société, nº50, p. 185-203.

Delaunay Bénédicte et al., 2010, «Chronique de l'administration», Revue française d'administration publique, vol. III, nº 135, p. 655-690.

DESRosIÈres Alain, 2008, L'argument statistique, I: Pour une sociologie historique de la quantification, Paris, Presses de l'École des mines.

Deumier Pascale, 2005a, «Les qualités de la loi », RTD Civ., p. 93 et suiv.

- 2005b, «Qu'est-ce qu'une loi ?», RTD Civ., p. 564 et suiv.

Drago Roland éd., 2005, La confection de la loi, Paris, PUF, Cahier des sciences morales et politiques.

Du MARAIS Bertrand, 2006a, "Les limites méthodologiques des rapports "Doing Business"», http://www.gip-recherche-justice.fr/aed/publications/limites_doing_ business_du_marais.pdf, consulté le 12 septembre 2012.

— 2006b, «Entre la Jamaïque et le Kiribati. Quelques réflexions sur l'attractivité du droit français dans la compétition économique internationale», Conseil d’État, Rapport public 2006, p. 377-389.

GILBERG Karine, 2007, La légistique au concret. Les processus de rationalisation de la loi, thèse de droit, Université Panthéon Assas.

GraSs Étienne, 2003, "L'inflation législative a-t-elle un sens? », Revue du droit public, $\mathrm{n}^{0} 1$, p. $139-162$.

LASSERRE Bruno, 2004, Pour une meilleure qualité de la réglementation, Paris, La Documentation française.

LOCHAK Danièle, 1993, «Quelle légitimité pour le juge administratif?», CURAPP, Droit et politique, Paris, PUF, p. 141-151.

MANDELKERn Dieudonné, 2002, La qualité de la réglementation, Paris, La Documentation française.

Mbongo Pascal, 2005, «De "l'inflation législative” comme discours doctrinal », Dalloz, chr., p. 1300 et suiv.

MerCIER Michel, 2000, Rapport d'information sur la sécurité juridique des actes des collectivités locales et les conditions d'exercice des mandats locaux, Rapport n ${ }^{0} 166$, Sénat.

MOYSAN Hervé, 2002, "La codification à droit constant ne résiste pas à l'épreuve de la consolidation », JCP-G, n² 27, 3 juillet, I, p. 147.

- 2007, «À propos de l'inflation des chiffres mesurant l'inflation des lois», Dalloz, p. 3029 et suiv.

- 2010, «De la mise en application de la loi à la qualité de sa rédaction. Quelques 
observations critiques sur des évolutions contrastées», JCP-A, $n^{\circ} 16,19$ avril, p. 2138 et suiv.

OGIEN Albert, 1995, L'esprit gestionnaire. Une analyse de l'air du temps, Paris, EHESS.

- 2010, «La valeur sociale du chiffre. La quantification de l'action publique entre performance et démocratie », Revue française de socio-économie, nº 5, p. 19-40.

PICQ Jean, 1995, L'État en France. Servir une nation ouverte sur le monde, Paris, La Documentation française.

QUILÈs Paul, 2006, Rapport tendant à modifier l'article 34 de la Constitution afin d'élargir les pouvoirs du Parlement, Rapport n³075, Assemblée nationale.

RENNES Juliette, 2011, «Les formes de la contestation. Sociologie des mobilisations et théories de l'argumentation », A contrario, vol. Il, nº16, p. 151-173.

RoLin Frédéric, 2006, «Le rapport 2006 du Conseil d’État, "Sécurité juridique et complexité du droit”. Quelques observations impertinentes», http://frederic-rolin. blogspirit.com (consulté le 11 juin 2007).

Rufin Michel, 1996, Rapport tendant à créer un office parlementaire d'amélioration de la législation, Rapport n 185 , Sénat.

SAVATIER René, 1977, "L'inflation législative et l'indigestion du corps social», Dalloz, chr., p. 43-48.

SECRÉTARIAT GÉNÉRAL DU GOUVERNEMENT, 2011, «Lois et règlements en vigueur. Approche statistique » : http://www.gouvernement.fr/sites/default/files/fichiers_joints/Lois_ et_reglements_en_vigueur_statistiques.pdf

Thomas Carole, 2008, Le bruit de la loi. Comment les lois deviennent médiatiques, thèse de sociologie, ENS Cachan.

VANNEUVILLE Rachel, 2010, «Le discours légistique du Conseil d’État. Mise en formes et usages politiques d'un discours critique sur le droit», P. Mbongo, O. Renaudie éd., Le Rapport public annuel du Conseil d'État. Entre science du droit et discours institutionnel, Paris, Cujas, p. 153-166.

VAUCHEZ Antoine, 2008, "Le chiffre dans le "gouvernement” de la justice», Revue française d'administration publique, nº 125, p.111-120. 